\title{
Radio Structures of Compact Quasars with Broad Absorption Lines
}

\author{
Magdalena Kunert-Bajraszewska ${ }^{1}$ and Marcin P. Gawroński ${ }^{1}$ \\ ${ }^{1}$ Toruń Centre for Astronomy, N. Copernicus University, Gagarina 11, 87-100 Toruń, Poland \\ Email: magda@astro.uni.torun.pl
}

\begin{abstract}
Broad absorption lines (BALs), seen in a small fraction of both the radio-quiet and radio-loud quasar populations, are probably caused by the outflow of gas with high velocities and are part of the accretion process. The presence of BALs is due to a geometrical effect and/or it is connected with the quasar evolution. Using the final release of FIRST survey combined with a catalog of BAL QSOs from SDSS/DR3, we have constructed a new sample of compact radio-loud BAL QSOs, which constitutes the majority of radio-loud BAL QSOs. The main goal of this project is to study the origin of BALs by analysis of the BAL QSOs radio morphology, orientation, and jet evolution using the European VLBI Network (EVN) at $1.6 \mathrm{GHz}$ and the Very Long Baseline Array (VLBA) at 5 and $8.4 \mathrm{GHz}$.
\end{abstract}

Keywords. galaxies: active, galaxies: evolution, (galaxies:) quasars: absorption lines

The radio morphologies of radio-loud BAL QSOs provide important additional information about their orientation and the direction of the outflow. However, most of them are compact at radio frequencies (Becker et al. 2000), so remained unresolved so far.

Using the final release of the FIRST survey combined with the Trump et al. 2006 catalog of BALs in SDSS Data Release 3, we have constructed a new sample (16 objects) of compact radio-loud BAL QSOs. They are also candidates for compact steep spectrum (CSS) and gigahertz-peaked spectrum (GPS) sources, which are considered to be young radio objects. The goals of the project are:

1. To study the origin of BALs by analysis the radio-loud BAL QSOs morphology. The high resolution radio observations will allow us to resolve their compact structures and to estimate the orientation of the jet axis.

2. To study the jet evolution and probable jet-cloud interactions. The BAL quasars can be still surrounded by the dense medium, since they are young radio sources like the CSS BAL quasar 1045+352 studied by Kunert-Bajraszewska \& Marecki (2007).

The first stage of the project was the $1.6 \mathrm{GHz}$ EVN observations of 10 of these 16 radio-loud BAL QSOs. All sources except one were detected and most of them remained unresolved, which shows they are indeed very compact objects. Some of them revealed core-jet structures, suggesting some beaming, but these must be confirmed by new observations at higher frequencies (VLBA 5 and $8.4 \mathrm{GHz}$ ) which will be published soon.

\section{Acknowledgements}

This work was supported by the Polish Ministry of Science and Higher Education under grant N N203 303635.

\section{References}

Becker, R. H., et al. 2000, ApJ, 538, 72

Kunert-Bajraszewska, M. \& Marecki, A. 2007, A 6 A, 469, 437

Trump, J. R., et al. 2006, ApJS, 165, 1 